\title{
Numerical Modeling of Liquid-Liquid Slug Flow in a Cross-Shaped Square Microchannel
}

\author{
Roman Filimonov ${ }^{1}$, Zan $\mathrm{Wu}^{2}$, Bengt Sundén ${ }^{2}$ \\ ${ }^{1}$ Lappeenranta-Lahti University of Technology LUT, School of Engineering Science \\ P.O. Box 20, Lappeenranta FI-53851, Finland \\ Roman.Filimonov@lut.fi \\ ${ }^{2}$ Lund University, Department of Energy Sciences \\ P.O. Box 118, Lund SE-22100, Sweden \\ Zan.Wu@energy.lth.se; Bengt.Sunden@energy.lth.se
}

\section{Extended Abstract}

Slug flow (or Taylor flow) in microchannels is a two-phase flow regime characterized by the motion of gas bubbles or liquid droplets in a continuous liquid flow. Heat and mass transfer from the fluid to the wall is improved due to internal recirculating flow within both the bubbles/droplets and the continuous phase liquid slugs which promotes the radial mixing of fluids. Slug flow is encountered in various applications of droplet-based microfluidic systems (e.g., DNA analysis, protein crystallization [1]). It is also used for microelectronics cooling [2,3]. While gas-liquid slug flow has been studied extensively, less research work, both experimental and numerical, has been done on liquid-liquid systems [2-4]. Wu et al. [5] have recently performed an experimental study on liquid-liquid slug hydrodynamics in square microchannels of cross-shaped junctions. However, it is difficult to make accurate measurements in microstructures. Furthermore, there are several factors influencing the slug hydrodynamics, such as flow rate, fluid viscosity, interfacial tension and channel geometry. Numerical modeling has emerged as a promising alternative way of studying microscale slug flow [6,7]. Numerical simulations can be utilized to study the effect of various parameters on slug dynamics, droplet and liquid slug sizes, heat transfer etc. Furthermore, modeling can provide flow details which are difficult or impossible to obtain directly from experiments. Thus, the present study aims to use computational fluid dynamics (CFD) to simulate the hydrodynamics of flow of a liquid (organic phase) dispersed in a continuous phase (aqueous phase) in a square channel of cross-shaped junction with a hydraulic diameter of $400 \mu \mathrm{m}$.

The Volume of fluid (VOF) method is employed to model the two-phase slug flow by tracking the liquid-liquid interface. Fluent 17.0 CFD software is used to perform the computations in the present study. Experimental results for water-butanol system are used to validate the numerical setup. Velocity field, liquid film thickness, distance between droplets, and droplet length, volume and velocity were extracted from the numerical simulations. The droplet formation process and droplet and water slug lengths were compared with the experimental ones. It was found that the three-phase contact angle (liquid-liquidsolid) affects the droplet formation frequency, hence the droplet size and the water slug length vary with the contact angle value applied. Therefore, the effect of the wall contact angle on the modeling of the droplet formation process is also investigated. The numerical setup will be also validated additionally using a different organic phase, e.g., toluene. Once the validation is done, the setup can be used to study the effects of liquid properties, channel geometry etc. on the slug hydrodynamics, and thus can help to obtain a better understanding of the related flow phenomena. In addition, as the numerical setup uses the same droplet generation mechanism as used in the experiments, it can be utilized to determine the flow rates of the liquids to generate droplets/water slugs of a desired size. New microchannel design can be developed by testing various options without involving experimental setups. The present model can be extended to study the heat transfer.

\section{References}

[1] H. Song, D. L. Chen, and R. F. Ismagilov, "Reactions in Droplets in Microfluidic Channels," Angewandte Chemie International Edition, vol. 45, no. 44, pp. 7336-7356, 2006.

[2] T. Bandara, N.-T. Nguyen, and G. Rosengarten, "Slug flow heat transfer without phase change in microchannels: A review," Chemical Engineering Science, vol. 126, pp. 283-295, 2015. 
[3] A. Abdollahi, R. N. Sharma, and A. Vatani, "Fluid flow and heat transfer of liquid-liquid two phase flow in microchannels: A review," International Communications in Heat and Mass Transfer, vol. 84, pp. 66-74, 2017.

[4] Z. Dai, Z. Guo, D. F. Fletcher, and B. S. Haynes, "Taylor flow heat transfer in microchannels-Unification of liquidliquid and gas-liquid results," Chemical Engineering Science, vol. 138, pp. 140-152, 2015.

[5] Z. Wu, Z. Cao, and B. Sundén, "Liquid-liquid flow patterns and slug hydrodynamics in square microchannels of crossshaped junctions," Chemical Engineering Science, vol. 174, pp. 56-66, 2017.

[6] R. Gupta, D. F. Fletcher, and B. S. Haynes, "On the CFD modelling of Taylor flow in microchannels," Chemical Engineering Science, vol. 64, no. 12, pp. 2941-2950, 2009.

[7] W. Lan, S. Li, and G. Luo, "Numerical and experimental investigation of dripping and jetting flow in a coaxial microchannel," Chemical Engineering Science, vol. 134, pp. 76-85, 2015. 